\title{
EFEITO DO SUBSTRATO NA EMERGÊNCIA, CRESCIMENTO E COMPORTAMENTO ESTOMÁTICO EM PLÂNTULAS DE MANGABEIRA ${ }^{1}$
}

\author{
REJANE JUREMA MANSUR CUSTÓDIO NOGUEIRA², MANOEL BANDEIRA DE ALBUQUERQUE ${ }^{3}$ JOSUÉ FRANCISCO \\ SILVA JUNIOR ${ }^{4}$
}

\begin{abstract}
RESUMO - Procedeu-se a um estudo em plântulas de mangabeira, com o objetivo de avaliar a germinação, crescimento inicial e relações hídricas em casa de vegetação. O delineamento experimental utilizado foi o inteiramente casualizado, com 50 repetições e três tipos de substratos: areia autoclavada, solo natural coletado em pomar espontâneo da espécie e uma mistura de húmus, areia e terriço vegetal na proporção de 2: 4: 4 (v/v/v). Verificaram-se a porcentagem de germinação, índice de velocidade de emergência (IVG), altura das plântulas, número de folhas, temperatura foliar (Tf), transpiração (E) e resistência difusiva (Rs). Foram mensuradas também a temperatura (Tar), umidade relativa (UR) e a radiação fotossinteticamente ativa (PAR). Os maiores percentuais de germinação e IVG ocorreram em areia autoclavada. A altura e o número de folhas não foram influenciados pelos substratos. Foram verificadas diferenças significativas entre os tratamentos para E e Rs. Folhas de plantas cultivadas em solo natural transpiraram mais do que as da mistura $\left(9,59 \mathrm{e} 2,59 \mathrm{mmol} \cdot \mathrm{m}^{-2} \cdot \mathrm{s}^{-1}\right.$, respectivamente), sendo que ambas diferiram daquelas cultivadas em areia autoclavada $\left(4,63 \mathrm{mmol} \cdot \mathrm{m}^{-2} \cdot \mathrm{s}^{-1}\right)$. A Rs em folhas no substrato areia foi de $2,2 \mathrm{~s} . \mathrm{cm}^{-1}$, enquanto, nas folhas em solo natural, esse valor foi de $0,9 \mathrm{~s} . \mathrm{cm}^{-1}$. Verificou-se efeito inverso e significativo para as correlações entre a Tf e E, e positivo entre Tf e Rs. Os efeitos de E x Rs foram inversos e altamente significativos para todos os tratamentos estudados. A Tf, E e Rs, bem como a altura das plântulas e o número de folhas não foram influenciados pela Tar, UR e PAR.
\end{abstract}

Termos para indexação: Hancornia speciosa, mangaba, crescimento, resistência difusiva, transpiração, fruticultura tropical.

\section{EFFECT OF THE SUBSTRATE ON THE EMERGENCE, GROWTH AND STOMATAL BEHAVIOR IN MANGABA SEEDLINGS}

\begin{abstract}
This study was carried out in greenhouse aiming to evaluate the germination, initial growth and water relations in mangaba seedlings. The experimental design was a completely randomized with fifty replicates and three substrates: autoclaved sand, local soil of the plants native region and a mixed of humus, sand and vegetal soil $(2: 4: 4, \mathrm{v} / \mathrm{v} / \mathrm{v})$. The following variables were studied: germination and emergence velocity (IVG) rates, seedling height, number of leaves, leaf temperature (LT), transpiration (E) and diffusive resistance (Rs). Also some climate variables were measured: air temperature (Tar), relative humidity (UR) and photosynthetic active radiation (PAR). The larger values of germination and IVG were observed in autoclaved sand substrate. Both plant height and number of leaves hadn't been influenced by substrates. There were significant differences among treatments in relation to $\mathrm{E}$ and Rs. Leaves of seedlings cultivated in native soil have transpired more than mixed substrate $\left(9.59\right.$ and $2.59 \mathrm{mmol} . \mathrm{m}^{-2} . \mathrm{s}^{-}$ ${ }^{1}$, respectively). Leaves cultivated in autoclaved sand showed intermediate value $\left(4.63 \mathrm{mmol} \cdot \mathrm{m}^{-2} \cdot \mathrm{s}^{-1}\right)$. There were variations on Rs of leaves for sand substrate and for native soil substrate (2.2 and $0.9 \mathrm{s.cm}$, respectively). Inverse and significant effect for correlations among LT and E was verified. Positive effect was observed among LT and Rs. Effects of E x Rs were inverse and significant for all treatments. LT, E and Rs, as well as seedling height and number of leaves were not influenced by Tar, UR and PAR.
\end{abstract}

Index terms: Hancornia speciosa, tropical fruit, diffusive resistance, transpiration.

\section{INTRODUÇÃO}

O Nordeste brasileiro vem destacando-se pelo elevado número de empresas de processamento de polpa de frutas, as quais têm mostrado extrema dificuldade na obtenção de matérias-primas que garantam o seu pleno funcionamento. Dentre as inúmeras espécies vegetais utilizadas para extração de polpa na região, destaca-se a mangabeira (Hancornia speciosa Gomes), planta de porte arbustivo que se encontra dispersa nas áreas de cerrado do Centro-Oeste e nas áreas de tabuleiros costeiros do Nordeste (Giacometti, 1993). Sua polpa presta-se para a fabricação de sorvetes, sucos, compotas e doces, além do látex que produz borracha e da madeira utilizada na carpintaria (Braga, 1960).

Por outro lado, a exploração de uma espécie nativa depende dos conhecimentos técnicos a respeito da propagação e do comportamento da mesma com relação às variações ambientais, os quais são escassos, principalmente aqueles ligados aos parâmetros fisiológicos frente às situações adversas. Dados referentes ao tipo de substrato são fundamentais no processo germinativo e estabelecimento da muda. Popinigis (1977) relata que o substrato apresenta grande influência na germinação, uma vez que fatores como estrutura, aeração, capacidade de retenção de água e grau de contaminação por patógenos podem variar segundo o material utilizado. Todas essas características são também decisivas no desenvolvimento e sobrevivência dessa espécie.

Alguns trabalhos foram realizados visando à definição do me- lhor substrato para cultivo de mangabeira. Vieira Neto (1998) indica o substrato areia e terra vegetal na proporção 1:1, 2:1 e 3:1, ou simplesmente terra vegetal como os mais adequados. No entanto, ainda são necessários estudos acerca da sua influência no comportamento fisiológico das plantas.

O presente estudo teve como objetivo avaliar o efeito do substrato sobre o processo germinativo, crescimento inicial e comportamento estomático em mangabeira cultivada em casa de vegetação.

\section{MATERIAL E MÉTODOS}

O experimento foi conduzido na casa de vegetação do Laboratório de Fisiologia Vegetal do Departamento de Biologia da Universidade Federal Rural de Pernambuco - UFRPE. As sementes foram provenientes de frutos coletados em estádio "de vez" no Sítio Santo Amaro, no município de Sirinhaém-PE ( $8^{\circ} 35^{\prime} 30^{\prime}$ 'S e $\left.35^{\circ} 07^{\prime} 00^{\prime} \mathrm{W}\right)$. Os frutos foram despolpados manualmente sob água corrente e as sementes imersas em hipoclorito de sódio a $2 \%$, por 5 minutos, e postas para secar à sombra, por um período de 20 horas. Logo em seguida, as mesmas foram semeadas a $1 \mathrm{~cm}$ de profundidade, em recipientes com capacidade de $150 \mathrm{~mL}$, contendo três tipos de substratos: areia autoclavada; uma mistura de húmus, areia de lavada e terriço vegetal na proporção de 2:4:4 (v/v/v), e solo natural coletado no mesmo local de origem das sementes. O referido solo é do tipo neossolo quartzarênico distrófico, bem drenado e de

\footnotetext{
${ }^{1}$ (Trabalho 154/2002). Recebido: 07/05/2002. Aceito para publicação: 09/04/2003.

${ }^{2}$ Bióloga, D.Sc., Professora Adjunto, Universidade Federal Rural de Pernambuco - UFRPE, Departamento de Biologia, Área de Botânica, bolsista CNPq, Rua Dom Manoel de Medeiros, s/n, Dois Irmãos, CEP 52171-900, Recife-PE. E-mail: rmansur@ hotlink.com.br;

${ }^{3}$ Eng. Agrônomo, Mestrando em Botânica, UFRPE, bolsista CNPq. E-mail: mbalbuquerque @ yahoo.com.br;

${ }^{4}$ Eng. Agrônomo, M.Sc., Embrapa Tabuleiros Costeiros, Cx. P. 44, CEP 49001-970, Aracaju-SE. E-mail: josué@ cpatc.embrapa.br
} 
textura arenosa. As análises físicas e químicas do solo da região de origem das sementes e do terriço que compunha a mistura estão apresentadas nas Tabelas 1 e 2 .

Os recipientes foram mantidos sob irrigação diária até a capacidade de pote, segundo metodologia de Nogueira (1997), sendo adotado o delineamento experimental inteiramente casualizado, com 50 repetições para cada tratamento. O período de avaliação da germinação foi de 30 dias após a semeadura, onde foi considerada germinada a plântula que emergisse o epicótilo. Também foi determinado o Índice de Velocidade de Emergência (IVG) nos diferentes substratos, segundo metodologia proposta por Popinigis (Santos \& Carlesso, 1998). Aos dois meses após a semeadu- ra, foram selecionadas cinco repetições (plantas) de cada tratamento e avaliados os parâmetros de crescimento: altura da plântula, utilizando-se de uma régua graduada, a partir do colo até a gema apical e o número de folhas. Foram medidas no período entre 12 e 13 horas (quando foi verificada maior abertura estomática), a transpiração (E), a resistência difusiva (Rs) e a temperatura foliar (Tf), na epiderme abaxial do terceiro par de folhas completamente expandidas, utilizando-se de um porômetro de equilíbrio dinâmico LICOR, modelo LI-1600. Paralelamente, a radiação fotossinteticamente ativa (PAR), a umidade relativa do ar (UR) e a temperatura do ar (Tar) foram monitoradas através de sensores quânticos e de umidade, e termopares acoplados ao referido porômetro.

TABELA 1 - Caracterização físico-química do solo natural de região de ocorrência de mangabeiras coletado numa profundidade de 0-20 cm, sob a copa de matrizes provenientes de sementes. Sítio Santo Amaro, Sirinhaém-PE.

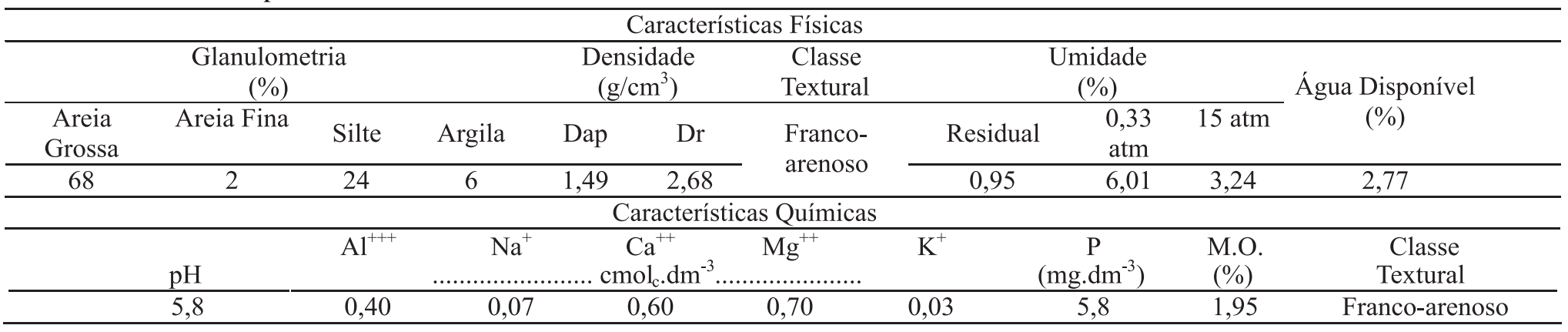

Dap: densidade aparente; Dr: densidade real

TABELA 2 - Caracterização físico-química do terriço vegetal coletado no câmpus da UFRPE na profundidade de 0-25 cm Recife-PE.

\begin{tabular}{|c|c|c|c|c|c|c|c|c|c|c|}
\hline \multicolumn{11}{|c|}{ Características Físicas } \\
\hline \multicolumn{4}{|c|}{ Glanulometria (\%) } & \multicolumn{2}{|c|}{ Densidade $\left(\mathrm{g} / \mathrm{cm}^{3}\right)$} & \multirow{3}{*}{$\begin{array}{c}\text { Classe Textural } \\
\text { Areia franca }\end{array}$} & \multicolumn{3}{|c|}{ Umidade $(\%)$} & \multirow[t]{2}{*}{ Água Disponível (\%) } \\
\hline Areia Grossa & Areia Fina & Silte & Argila & Dap & $\mathrm{Dr}$ & & Residual & $0,33 \mathrm{~atm}$ & $15 \mathrm{~atm}$ & \\
\hline 70 & 13 & 9 & 8 & 1,50 & 2,72 & & 0,90 & 14,87 & 9,99 & 4,88 \\
\hline \multicolumn{11}{|c|}{ Características Químicas } \\
\hline \multirow{2}{*}{\multicolumn{2}{|c|}{$\mathrm{PH}$}} & \multirow{2}{*}{\multicolumn{2}{|c|}{$\mathrm{Al}^{+++}$}} & $\mathrm{Na}^{+}$ & & $\mathrm{Ca}^{++}$ & $\mathrm{Mg}^{++}$ & $\mathrm{K}^{+}$ & & $\mathrm{P}$ \\
\hline & & & & $\ldots$ & $\ldots \ldots$ & ......cmolc/dm $3 \ldots$. & ........ & ..................... & & $\left(\mathrm{mg} / \mathrm{dm}^{3}\right)$ \\
\hline \multicolumn{2}{|l|}{6,0} & \multicolumn{2}{|c|}{0,00} & - & & 6,25 & 1,35 & 0,20 & & 40 \\
\hline
\end{tabular}

Dap: densidade aparente; Dr: densidade real

Foi realizada análise de variância e comparação de médias pelo teste de Tukey. Além disso, foram obtidas correlações simples entre as variáveis.

\section{RESULTADOS E DISCUSSÃO}

Os maiores percentuais de germinação e valores de IVG foram obtidos no substrato areia ( $68 \%$ e 5,28, respectivamente), provavelmente por reunir características necessárias de um bom substrato para germinação, tais como porosidade e esterilidade. Uma boa porosidade permite o movimento de água e ar no substrato, favorecendo a germinação. Para que isso ocorra, as sementes não necessitam de nutrientes, mas apenas de sua hidratação e aeração para que se procedam as reações que induzam à formação do caulículo e radícula. A esterilidade do substrato seria outro fator importante para o aumento na taxa de germinação das sementes, não servindo como fonte de patógenos de solo que poderiam afetar a germinação e o estabelecimento das plântulas (Simão, 1971). Os valores de porcentagem de germinação para os substratos solo natural e areia foram similares aos encontrados por Parente \& Machado (1986), mas diferentes para o substrato mistura (Tabela 3 ). Semelhantemente ao ocorrido na germinação, o IVG dos demais tratamentos variou de 2,82 no solo natural e 1,30 na mistura. Segundo Vieira Neto \& Carvalho (1994), o tamanho da semente é um fator complicador nesse tipo de teste, pois as sementes menores, por necessitarem de menor volume de água, apresentam uma rápida germinação. Em contrapartida, as de maior tamanho, por possuírem maiores conteúdos de reserva, originam plântulas de maior tamanho. Por outro lado, Araújo \& Nogueira (2000) reportaram que o tamanho das sementes em mangabeira não deve ser utilizado como uma característica indicativa de viabilidade de embriões, não sendo, portanto, recomendável como critério para implantação de um programa de seleção de sementes, visando à produção de mudas.

TABELA3- Valores médios da porcentagem de germinação (\%G) e índice de velocidade de germinação (IVG) de sementes de mangaba cultivadas em diferentes substratos*.

\begin{tabular}{lcc}
\hline Substratos & $\% \mathrm{G}$ & IVG \\
\hline Solo natural & $56 \mathrm{a}$ & $2,82 \mathrm{ab}$ \\
Areia autoclavada & $68 \mathrm{a}$ & $5,28 \mathrm{a}$ \\
Mistura & $28 \mathrm{~b}$ & $1,30 \mathrm{~b}$ \\
\hline
\end{tabular}

* Letras iguais, na coluna, não diferem estatisticamente entre si, pelo teste de Tukey, a 5\% de probabilidade.

As plântulas do solo natural apresentaram uma altura média ligeiramente superior $(6,35 \mathrm{~cm})$ àquelas dos demais tratamentos, embora não tenha ocorrido diferença significativa entre os mesmos. Em relação ao número de folhas, também não foram constatadas diferenças estatísticas, havendo uma variação de 6,0 (solo natural), 6,7 (mistura) a 7,5 (areia lavada), conforme observado na Figura 1. 


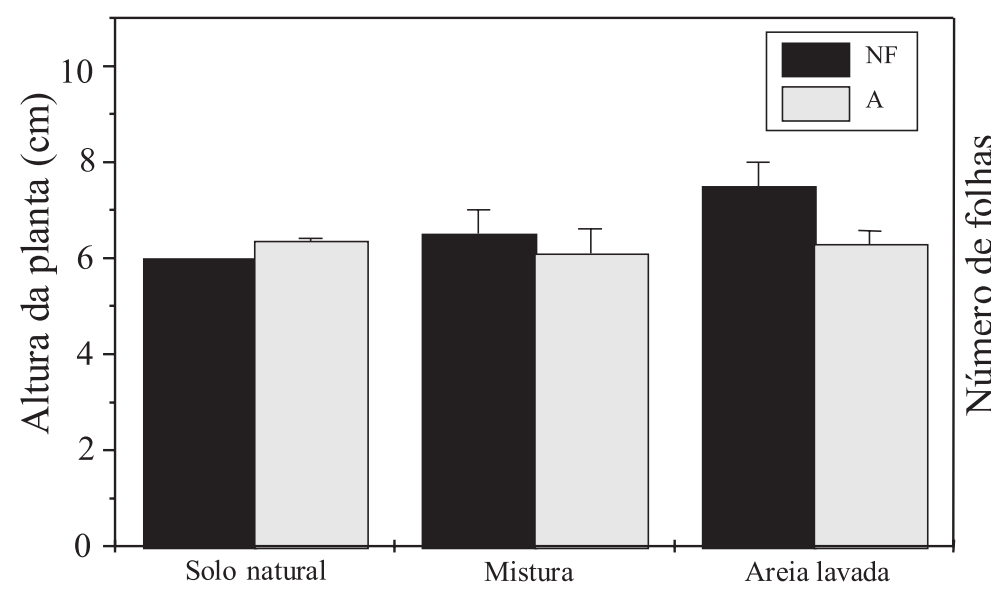

Tipos de substratos

FIGURA 1-Valores médios da altura (A) e número de folhas (NF) em plântulas de mangabeira cultivadas em casa de vegetação, em diferentes tipos de substrato.

Os valores das variáveis climáticas (PAR, UR, Tar e DPV), no interior da casa de vegetação, entre 12 e $13 \mathrm{~h}$ variaram de 785,0 a 869,0 $\mu$ mol. $\mathrm{m}^{-2} \mathrm{~s}^{-1} ; 47,0$ a $48,0 \% ; 36,4$ a $36,7^{\circ} \mathrm{Ce} 3,16$ a $3,27 \mathrm{kPa}$, respectivamente.

A análise de variância para a transpiração (E) e resistência difusiva (Rs) apresentou diferenças altamente significativas entre os tratamentos, exceto para a temperatura foliar. A Rs variou de 0,9 a 4,0 $\mathrm{s} . \mathrm{cm}^{-1}$, sendo que o menor valor foi verificado em solo natural, enquanto o maior ocorreu em folhas de plantas cultivadas na mistura com valor intermediário para areia autoclavada (Figura 2). Em estudo realizado por Nogueira et al. (1999), folhas de mangabeira expostas ao sol apresentaram variação de 1,1 a 4,5 s.cm-1 de Rs. Com relação a esse parâmetro, observaram-se comportamentos distintos entre os tratamentos, destacando- se o solo natural, o qual proporcionou maior grau de abertura estomática, mantendo no horário de maior demanda evaporativa os menores valores de Rs (Figura 2), possibilitando com isso uma maior absorção de $\mathrm{CO}_{2}$ para a fotossíntese. Oliveira et al. (1995) estudaram o comportamento de plantas jovens de algumas espécies frutíferas tropicais e subtropicais e constataram respostas fisiológicas diferenciadas quando submetidas a diferentes níveis de radiação solar. Segundo esses autores, plantas de carambola (Averrhoa carambola L.), cupuaçu (Theobroma grandiflorum Schum.) e biribá (Rollinia sp.), cultivadas à sombra, apresentaram maior condutância estomática do que quando cultivadas em pleno sol. Por outro lado, para araticum (Annona sp.), jenipapo (Genipa americana L.) e murici (Byrsonima sp.) cultivados nas mesmas condições, a resposta foi inversa, ou seja, a condutância manteve-se maior em plantas expostas à radiação solar direta.

Os maiores valores da transpiração em folhas de mangabeira foram verificados em solo natural $\left(9,6 \mathrm{mmol} \cdot \mathrm{m}^{-2} \cdot \mathrm{s}^{-1}\right)$, enquanto os menores ocorreram em plantas cultivadas na mistura $\left(2,6 \mathrm{mmol} \cdot \mathrm{m}^{-2} \cdot \mathrm{s}^{-1}\right)$. Para folhas de plantas cultivadas em areia, os valores foram de 4,6 mmol. $\mathrm{m}^{-2} . \mathrm{s}$ ${ }^{1}$ (Figura 2).

Trabalhando com quatro matrizes de mangabeira na Zona da Mata Sul de Pernambuco, Nogueira et al. (1999) verificaram padrões de comportamento estomático diferentes para as referidas matrizes. No horário de maior demanda evaporativa, os genótipos M-UFRPE-1 e MUFRPE-8 conseguiram manter as trocas gasosas com mais eficiência, apresentando valores da transpiração iguais a 6,6 e $8,5 \mathrm{mmol} . \mathrm{m}^{-2} . \mathrm{s}^{-1}$, respectivamente, quando comparadas à matriz M-UFRPE-2, a qual transpirou nesse mesmo horário $2,2 \mathrm{mmol} \cdot \mathrm{m}^{-2} \cdot \mathrm{s}^{-1}$. Comparando-se esses valores com os obtidos no presente trabalho, verifica-se que a taxa transpiratória das folhas de mangabeira, cultivada em solo natural, nas horas mais quentes do dia, é relativamente alta, podendo dessa forma facilitar a entrada do $\mathrm{CO}_{2}$ para a realização da fotossíntese e proporcionar maior crescimento das plantas.

Os valores da temperatura das folhas mantiveram-se superiores à temperatura do ar em todos os tratamentos, com uma diferença máxima de $0,37^{\circ} \mathrm{C}$ (Figura 2). Em plantas adultas cultivadas no campo,

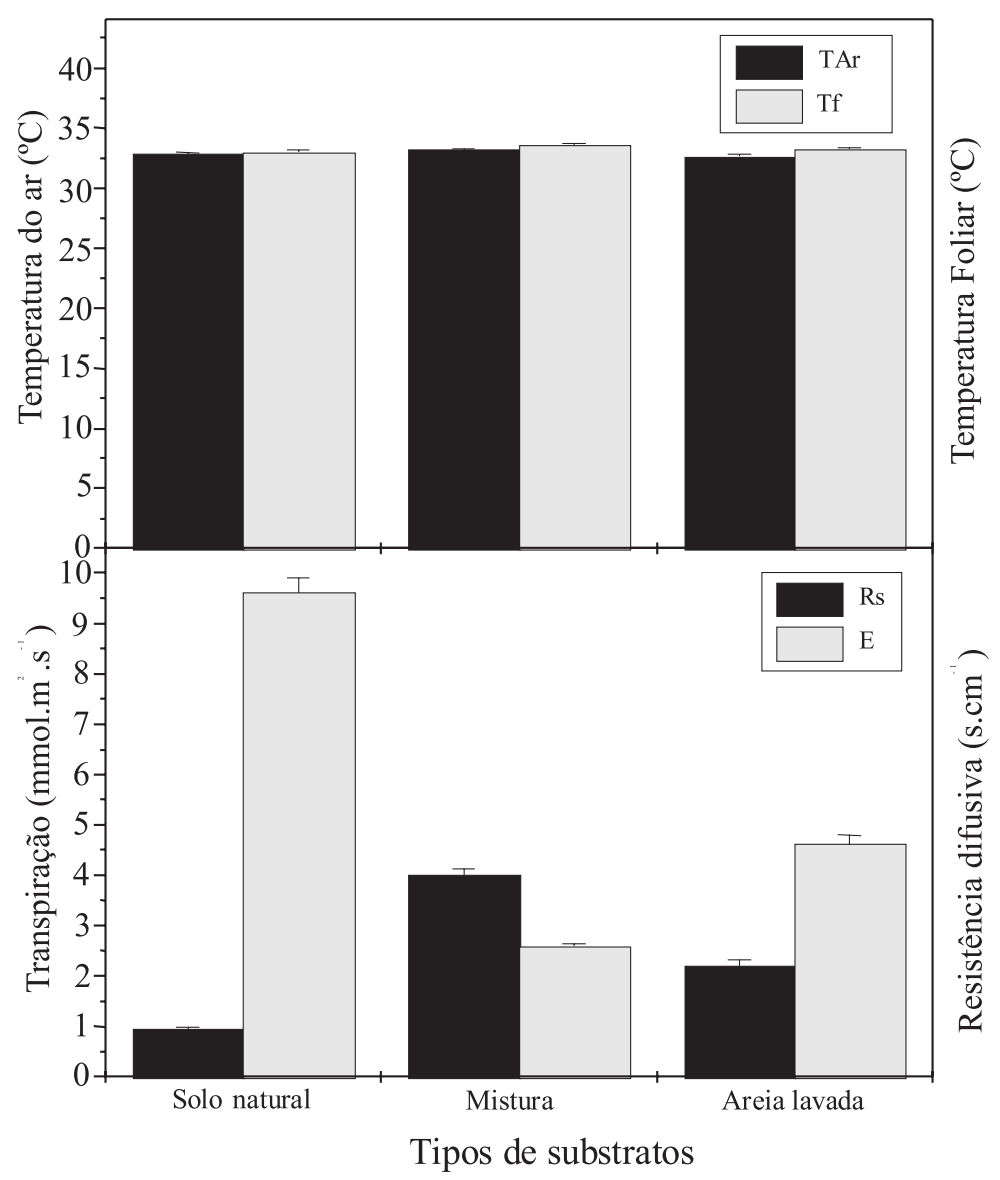

FIGURA 2-Valores médios da transpiração (E), resistência difusiva (Rs), temperaturas do ar (Tar) e folha (Tf) em plântulas de mangabeira cultivadas em casa de vegetação, em diferentes tipos de substrato.

Nogueira et al. (1999) observaram que a temperatura das folhas esteve acima da temperatura do ar, com uma diferença máxima de $2,5^{\circ} \mathrm{C}$, verificada no genótipo M-UFRPE 2 e mínima de $1^{\circ} \mathrm{C}$ para M-UFRPE 3. Segundo os mesmos autores, as folhas sombreadas apresentaram suas temperaturas inferiores às registradas para o ambiente próximo a elas, com uma variação de 0,2 a $0,7^{\circ} \mathrm{C}$. Resultados semelhantes foram verificados em folhas de laranjeira (Citrus sinensis) (Machado et al., 1994) e de acerola (Malpighia emarginata D.C.), durante a estação seca (Nogueira \& Moraes, 1997). Os primeiros autores relatam que as temperaturas das folhas expostas à radiação solar estiveram entre $1^{\circ} \mathrm{C}(9 \mathrm{~h})$ e $5^{\circ} \mathrm{C}(13 \mathrm{~h})$ acima da temperatura do ar, enquanto os segundos verificaram diferenças de até $4^{\circ} \mathrm{C}$. Essa variação é comum em algumas espécies cultivadas e fundamenta-se no estado hídrico das mesmas, no comportamento estomático e na perda de calor latente através da transpiração. Tais diferenças normalmente diminuem no início da manhã e final da tarde (Salinas et al., 1996).

Observando-se o sistema radicular de algumas plântulas após as análises porométricas, no presente trabalho, foi detectada a presença de fungos micorrízicos arbusculares (FMA's) principalmente nas raízes de plântulas cultivadas em solo natural. Várias pesquisas têm sido conduzidas sobre a influência de FMAs nas plantas, principalmente no que diz respeito ao aumento de resistência a organismos fitopatogênicos (Chu et al., 1997; Oliveira et al., 1999). Os FMAs promovem alterações na morfologia, fisiologia ou bioquímica da planta hospedeira (Oliveira et al., 1999). As morfológicas são a lignificação da parede celular, produção de diversos polissacarídeos e o aumento do sistema radicular, sendo este último fator responsável pelo aumento do fluxo de água e nutrientes pela planta. Andrade et al. (1999) sugerem que a micorriza seja um fator importante na sobrevivência de mudas de mangaba em solos de baixa fertilidade. $\mathrm{O}$ estudo feito por esses autores revela que o incremento excessivo de matéria orgânica às mudas de mangabeira pode favorecer o desenvolvimento de patógenos nas raízes, o que explicaria a baixa 
transpiração das plântulas do substrato com mistura de areia, terriço vegetal e húmus, apesar de estar sob capacidade de pote.

Verificou-se efeito inverso e significativo para as correlações entre a Tf e $\mathrm{E}\left(-0,6306^{*}\right)$, e positivo e significativo entre Tf e Rs $\left(0,6712^{*}\right)$. Os efeitos de $\mathrm{E} x$ Rs foram inversos e altamente significativos $\left(-0,9371^{* *}\right)$ para todos os tratamentos estudados. A Tf, E e Rs, bem como a altura das plântulas e número de folhas, não foram influenciadas pela Tar, UR e PAR.

\section{CONCLUSÕES}

1) O substrato areia autoclavada é o que proporciona maior porcentagem de germinação e IVG dentre os substratos utilizados.

2) As plântulas cultivadas no substrato solo natural apresentam maior transpiração que as cultivadas nos demais substratos.

3) Plantas cultivadas em solo natural apresentam maior adaptação ao ambiente devido à diminuição da resistência difusiva, favorecendo as trocas gasosas nas horas de maior demanda evaporativa.

\section{REFERÊNCIAS BIBLIOGRÁFICAS}

ANDRADE, L.R.M.; JUNQUEIRA, N.T.V.; SILVA, J.A.; BARBOSA, D.; LEÃO, A.P.; BARROS, L.H. Fertilização do substrato e inoculação de fungos micorrízicos arbusculares em mudas de mangaba (Hancornia speciosa Gomes.). In: CONGRESSO BRASILEIRO DE CIÊNCIADO SOLO, 27, 1999, Brasília. Anais... Brasília: Embrapa Cerrados/SBCS, 1999 (Disponível do CD-ROM).

ARAÚJO, E.L.; NOGUEIRA, R.J.M.C. Tamanho de sementes e viabilidade de embriões em mangabeira (Hancornia speciosa Gomes). Naturalia, São Paulo, v. 25, p. 139-148. 2000.

BRAGA, R. Plantas do Nordeste, especialmente do Ceará. 4.ed. Natal: Universitária UFRN, 1960. 540p.

CHU, E.Y.; ENDO, T.; STEIN, R.L.B.; ALBUQUERQUE, F.C. Avaliação da inoculação de fungos micorrízicos arbusculares sobre a incidência da fusariose da pimenta-do-reino. Fitopatologia Brasileira, Brasília, n. 22, p. 205-208, 1997.

GIACOMETTI, D.C. Recursos genéticos de fruteiras nativas do Brasil. In: SIMPÓSIO NACIONAL DE RECURSOS GENÉTICOS DE FRUTEIRAS NATIVAS, 1992, Cruz das Almas. Anais... Cruz das Almas: Embrapa-CNPMF, 1993, p.13-27.

MACHADO, E.C.; QUAGGIO, J.A.; LAGÔA, A.M.M.A.; TICELLI, M.; FURLANI, P.R. Trocas gasosas e relações hídricas em laranjeiras com clorose variegada dos cítrus. Revista Brasileira de Fisiologia
Vegetal, Brasília, v. 6, n. 1, p. 53-57, 1994.

NOGUEIRA, R.J.M.C.; MORAES, J.A.P.V. Ecofisiologia da acerola I. Transpiração, resistência difusiva e temperatura foliar. In: SEMINÁRIO REGIONAL DE ECOLOGIA, 8, 1996, São Carlos. Anais... São Carlos: Programa de Ecologia e Recursos Naturais - UFSCar, 1997, v.3, cap.1, p.1085-1091.

NOGUEIRA, R.J.M.C.; MELO FILHO, P.A.; ARAÚJO, E. Expressões ecofisiológicas de germoplasma de Hancornia speciosa Gomes cultivado no litoral pernambucano. Ciência Rural, Santa Maria, v. 29, n. 4, p. 731-732, 1999.

NOGUEIRA, R.J.M.C. Expressões fisiológicas da aceroleira (Malpighia emarginata D.C.) em condições adversas. São Carlos-SP: UFSCar, 1997. 207p. (Tese de Doutorado).

OLIVEIRA, A.A.R.; COELHO, Y.S.; COUTO, R.C.S. Relação entre fungos micorrízicos arbusculares e incidência de declínio em lima ácida 'Tahiti'. Revista Brasileira de Fruticultura, Jaboticabal, v. 21, n. 3, p. 342-345, 1999 .

OLIVEIRA, GM.B.;ALBUQUERQUE, M.B.; NOGUEIRA, R.J.M.C.;ARAÚJO, E.L. Avaliação da germinação e crescimento inicial de Hancornia speciosa Gomes. In: CONGRESSO DE INICIAÇÃO CIENTIFICADA UFRPE, 8, 1998, Recife. Anais... Recife: Imprensa Universitária, 1998, p. 102

PARENTE, T.V.; MACHADO, J.W.B. Germinação de sementes de mangaba (Hancornia pubescens Nees e Mart.) provenientes de frutos colhidos com diferentes graus de maturação. Revista Brasileira de Fruticultura, Cruz das Almas, v.1, n. 8, p. 39-43, 1986.

POPINIGIS, F. Fisiologia da semente. Brasília: AGIPLAN, 1977. 289p.

SALINAS, A.P.R.; ZELENER, N.; CRAVIOTTO, R.M.; BISARO, Z. Respuestas fisiologicas que caracterizan el comportamiento de diferentes cultivares de soja a la diferencia hidrica em el suelo. Pesquisa Agropecuária Brasileira, Brasília, v. 31, n. 5, p. 331-338, 1996.

SANTOS, R.F.; CARLESSO, R. Déficit hídrico e os processos morfológico e fisiológico das plantas. Revista Brasileira de Engenharia Agrícola e Ambiental, Campina Grande, v.2, n. 3, p. 287-294, 1998.

SIMÃO, S. Manual de Fruticultura. São Paulo: Ceres, 1971. 530p.

VIEIRA NETO, R.D. Efeitos de diferentes substratos na formação de mudas de mangabeira (Hancornia speciosa Gomes). Revista Brasileira de Fruticultura, Jaboticabal, v. 20, n. 3, p. 265-271, 1998.

VIEIRA, R.D.; CARVALHO, N.M. Testes de vigor baseados na avaliação de plântulas. In: NAKAGAWA, J. (Ed.) Testes de vigor em sementes. Jaboticabal: FUNEP, 1994. p. 49-86. 